\title{
Different Types of Noise Removal in Magnetic Resonance Imaging (MRI) Image using Threshold Values
}

\author{
Awani Tiwari \\ M. Tech. Scholar \\ Department of Electronics and Communication \\ BIST, Bhopal
}

\author{
K. P. Singh \\ Assistant Professor \\ Department of Electronics and Communication \\ BIST, Bhopal
}

\begin{abstract}
In an advanced imaging technique Magnetic Resonance Imaging (MRI) plays a major role in medical field to create high standard images contained in the human brain. MRI imaging is often used when treating brain, prostate cancers, ankle and foot. Noise elimination is the main constraint in digital image processing and sometimes it is very difficult to find out the origin of the noise.
\end{abstract}

\section{Keywords}

MRI image, various noise, PSNR, MSE

\section{INTRODUCTION}

Presently multi day, a large portion of the data in Computer preparing is taken care of on the web. This online data is either graphical or pictorial in nature, and the capacity and correspondence prerequisites are tremendous. Thus technique for packing the information before capacity and transmission are of critical useful and business intrigue. Picture pressure implies diminishing the repetitive measure of information required to speak to an advanced picture. The Digital picture pressure in numerical structure can be characterized as change of a 2-D pixel exhibit by picture, into a factually uncorrelated informational collection. The change is connected on picture before capacity and transmission of Digital Image Data. The packed picture is recreated into unique picture by the procedure of Decompression. Decompressed picture can be a unique picture or estimation of it. Picture pressure is the innovation for dealing with the expanded spatial goals of the present imaging sensors and developing communicate TV norms. Picture pressure assumes an essential job in numerous critical and assorted applications including tele video conferencing, remote detecting, archive and medicinal imaging, copy transmission and the control of remotely guided vehicles in military, space, and risky waste administration applications. The application list is consistently developing the effective control stockpiling and transmission of various kinds of computerized picture, for example, paired pictures, dim scale pictures, and shading pictures and so forth [1], [2] The Internet, still in its youth; keeps on prospering and effect on our own and expert lives. Regular to these and numerous different applications is the prerequisite of immense extra room and correspondence transmission capacity for computerized pictures. Subsequently computerized media is persuaded by creative techniques for pressure of advanced pictures for productive use of extra room and correspondence data transfer capacity [3], [4]. As a rule setting, the picture talking pressure systems can be partitioned into two expansive classes: lossless pressure and lossy pressure plans. Lossless Compression (Information protecting): As the name suggests, this procedure includes no loss of information. The first information can be recuperated precisely from the compacted information.
A CT check are on sort of unique $\mathrm{x}$-beam tests which is produce cross-sectional pictures of the body utilizing PC and $\mathrm{x}$-beams it connects a noteworthy job diagnosing medicinal infections, it is utilized to know subtleties of human body like chest, paunch, pelvis, arm, leg, by utilizing CT filter pictures of organ like liver, pancreas, digestive system, kidney, bladder, adrenal organ, lung and heart, and so on. The MRI is a systems to get a reasonable picture of organs by utilizing extensive measure of attractive and radio waves. It uses to analyze an assortment of conditions from tendons to tumors and will be utilized to think about mind and spinal line. In restorative picture handling Denoising of pictures assumes an imperative job to get exact and precise pictures for further finding. Therapeutic pictures are gathered by various sensors and they are likewise oppressed wide assortment of mutilation, stockpiling, pressure, procurement, preparing, propagation And transmission which makes them get defiled by various kinds of clamors are evacuated utilizing channels as they can create best outcomes relying on its parameters. The determination of channels rely on they kind of commotion on the grounds that distinctive sort of clamor can be evacuated utilizing diverse kinds of commotions. In this paper a noised picture is considered and it is sifted utilizing Median and Wiener channel and the outcome is looked at on different parameters. Middle channel and Wiener channel calculation will be changed. Different clamors and like salt and pepper commotion are included. Wiener channel and middle channel are actualized to evacuate added substance commotion which is available in MRI and CT filters which likewise capable to include thickness step by step. Superconductive scanner contains refrigeration framework and fluid helium siphon which is in charge of "pound" sound, which is additionally aggravate patient and leads brief hoop misfortune.

\section{MRI IMAGE}

X-rays utilize amazing magnets which produce a solid attractive field that powers protons in the body to line up with that field. At the point when a radiofrequency current is then beat through the patient, the protons are invigorated, and turn out of balance, stressing against the draw of the attractive field. 


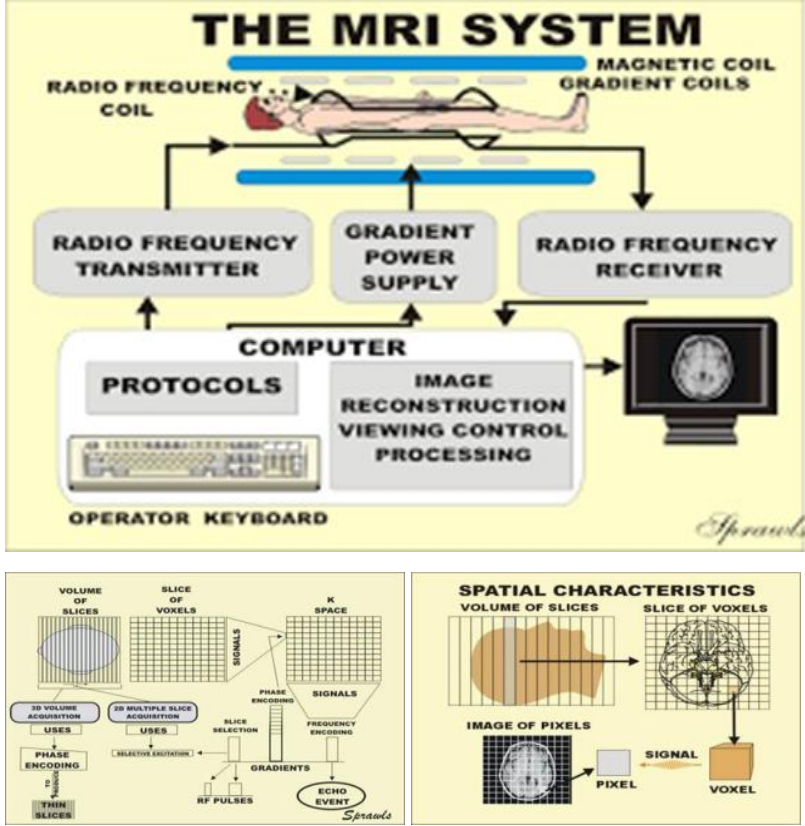

Figure 1: Working of MRI Image

At the point when the radiofrequency field is killed, the MRI sensors can identify the vitality discharged as the protons realign with the attractive field.

Attractive polarization .Very solid uniform magnet excitation .Very amazing rf transmitter Acquisition, Location is encoded by inclination attractive fields .Very ground-breaking audi amps Polarization, Proton have an attractive minute proton have turns like pivoting magnets Body has a ton of protons.

\section{MEDIAN FILTER}

In sign preparing, it's far frequently appropriate that enables you to do some sort of commotion decrease on a photo or sign. The center filter through is a nonlinear progressed isolating framework, sometimes used to empty bustle. Such uproar refund is a typical pre-taking care of dare to upgrade the consequences of later getting ready (for example, side area on an image). Center filtering could be commonly used as a piece of automated photograph preparing because of the reality, underneath specific circumstances, it jelly edges even as getting rid of commotion (anyway observe discourse under).The rule thought of the center get out is to experience the sign area through get to, replacing each section with the center of neighboring entries. The case of partners is known as the "window", which slides, access with the guide of access, over the all-out banner. For 1D sign, the most outrageous clear window is basically the basic couple of past and taking after areas, while for $2 \mathrm{~d}$ (or higher-dimensional) alerts including photographs, progressively complicated window models are sensible (which join "holder" or "go" structures). Realize that in the event that the window has an odd wide arrangement of entries, at that point the center is definitely not hard to describe: it is basically within regard after all of the segments inside the window is arranged numerically. For an even wide grouping of sections, there is a few reasonable center, see center for extra information.

See that, for the situation above, in light of the way that there is no get to past the main regard; the basic cost is repeated, likewise with an authoritative charge, to get sufficient entries to fill the window. This is one strategy for adjusting to lacking window entries at the obstacles of the banner, yet there are various plans that have exceptional houses that might be liked especially precedents:
- Avoid dealing with the points of confinement, with or without cutting the banner or picture limit some time later,

- Fetching segments from better places in the banner. With pictures for example, areas from the far level or vertical farthest point might be picked,

- Shrinking the window near the points of confinement, with the goal that every window is full.

\section{TYPES OF NOISE}

Commotion is included inside the image at the season of photo obtaining or transmission. Diverse variables might be responsible for presentation of clamor inside the photograph. The wide assortment of pixels tainted in the image will choose the measurement of the commotion. The central resources of commotion inside the virtual picture are:

a) The imaging sensor might be stricken by ecological conditions at some phase in picture obtaining.

b) Inadequate mellow degrees and sensor temperature may furthermore present the commotion in the image.

c) Interference in the transmission channel may likewise degenerate the photograph.

d) If earth flotsam and jetsam is blessing at the scanner show screen, they additionally can present commotion inside the photo.

Commotion is the bothersome outcomes delivered inside the image. For the span of photograph procurement or transmission, various components are chargeable for presenting commotion in the photograph. Depending at the kind of unsettling influence, the commotion can affect the image to exceptional volume. Usually our discernment is to push off beyond any doubt sort of commotion.

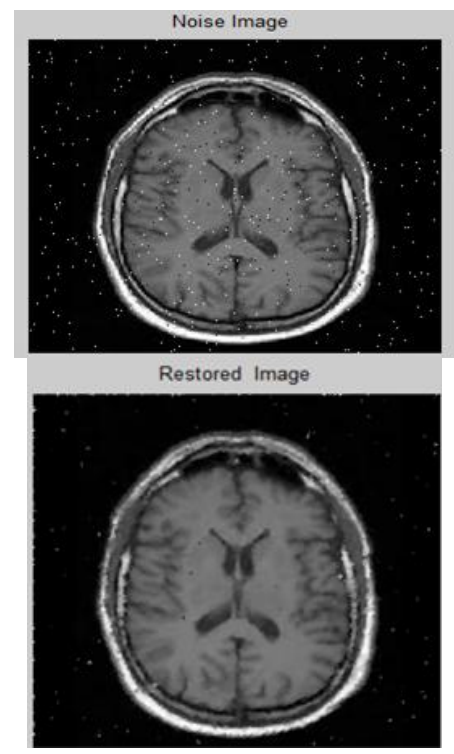

Figure 2: Salt and Pepper noise and original image

So we become mindful of beyond any doubt sort of upheaval and apply unique computation to dispose of the fuss. Picture tumult can be arranged as Impulse fuss (Salt-and-pepper clatter).

\section{Gaussian Noise}

Gaussian dispersion which is otherwise called typical circulation whose Probability Density Function is equivalent to factual clamor known as Gaussian Noise. This clamor is expelled from the computerized pictures by smoothening of the picture pixels which helps in lessening the force of the commotion present in the picture which is caused because of securing however the outcome perhaps at some point bothersome and furthermore 
which can bring about obscuring edges of the astounding pictures [2].

\section{Spot Noise}

The Speckle Noise is characterized as a commotion which is available in the pictures and which corrupts the nature of a picture. Dot Noise is a marvel that escorts all cognizant imaging modular quality in which pictures are delivered by meddling echoes of a transmitted waveform that start from assorted variety of the considered articles [5].These are the granular commotions that are in a general sense present in the picture and lessen the nature of the dynamic radar and Synthetic Aperture Radar (SAR) pictures or Magnetic Resonance [6]. Imaging (MRI) pictures is alluded to as Speckle Noise. In the event that Speckle Noise is available in the ordinary radar results from irregular varieties in the arrival motion from an article which is never again picture process flag builds the mean dark dimension in a picture. A Speckle Noise is the cognizant imaging of items in the picture. Indeed, it is caused because of mistakes in information transmission. This sort of commotion influences the ultrasound pictures and MRI pictures.

\section{PROPOSED METHODOLOGY}

The Modified Median Filter figuring is the time when a picked window contains only 0 and 255 regard then the restored regard is either 0 or 255(again uproarious), drives us to proposed. In this count we picked pixel regard 0 and 255 qualities then the getting ready pixel is replaced by mean estimation of the picked window. The detail of the figuring is given underneath.

\section{Calculation:}

Stage 1: Select a 3 x 3 grid size as indicated by the 2-D window measure. Expect that the handling pixel is Pij, which lies at the focal point of window.

Stage 2: If $0<\mathrm{Pij}<255$, at that point the handling pixel or $\mathrm{Pij}$ is uncorrupted and left unaltered.

Stage 3: in the unlikely event that $\mathrm{Pij}=0$ or $\mathrm{Pij}=255$, at that point it is considered as corrupted pixel and four cases are possible as given underneath.

Case 1: if the picked window has all the pixel regard as 0, at that point Pij is superseded by the Salt fuss (for example 255).

Case 2: if, despite everything that the picked window contains all the pixel regard as 255 , at that point $\mathrm{Pij}$ is superseded by the pepper upheaval (for example 0 ).

Case 3: if the picked window contains all the regard as 0 and 255 both. By then the dealing with pixel is superseded by mean estimation of the window.

Case 4: if, despite everything that the picked window contains not all the segment 0 and 255. By then discard 0 and 255 and find the center estimation of the remainder of the part. Displace Pij with center regard.

Stage 4: Rehash stage 1 to 3 for the entire picture until the methodology is done.

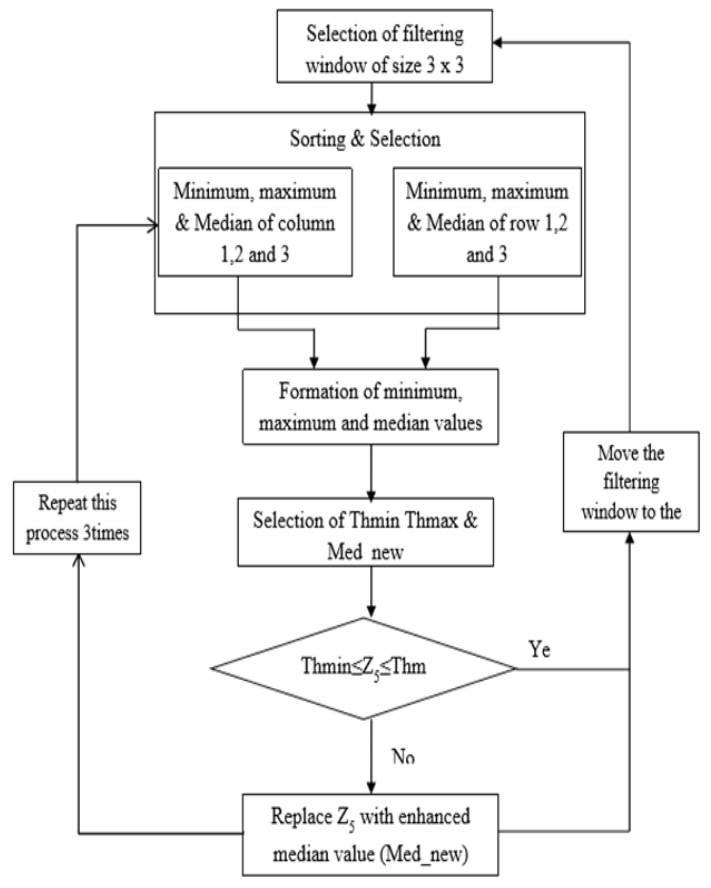

Figure 3: Flow Chart of Proposed Method

\section{SIMULATION RESULT}

The proposed calculations are tried utilizing 256x256 8bit/pixel picture bike.jpg. In the reproduction, pictures are tainted by Salt and Pepper commotion. The commotion level shifts from 10\% to $90 \%$ with augmentation of $10 \%$ and the execution is quantitatively measured by Mean square Error (MSE) and Peak Signal to Noise Ratio (PSNR).

Normalized Absolute Error (NAE)

$$
=\frac{1}{N_{1} N_{2}} \sum_{j=1}^{N_{2}} \sum_{i=1}^{N_{1}}(f(i, j)-g(i, j))
$$

Mean Square Error (MSE)

$$
=\frac{1}{N_{1} N_{2}} \sum_{j=1}^{N_{2}} \sum_{i=1}^{N_{1}}(f(i, j)-g(i, j))^{2}
$$

Root Mean Square Error (MSE)

$$
=\sqrt{M S E}
$$

Peak Signal to Noise Ratio (PSNR) in $\mathrm{dB}$

$$
=10 \times \log _{10}\left(\frac{255^{2}}{M S E}\right)
$$

Where MSE remains for Mean Square Error, PSNR remains for Peak Signal to Noise Ratio. From the reproduction result appeared in Table I to II, it is watched that the execution of proposed calculation is enhanced PSNR than the current calculations at medium and high clamor level. 

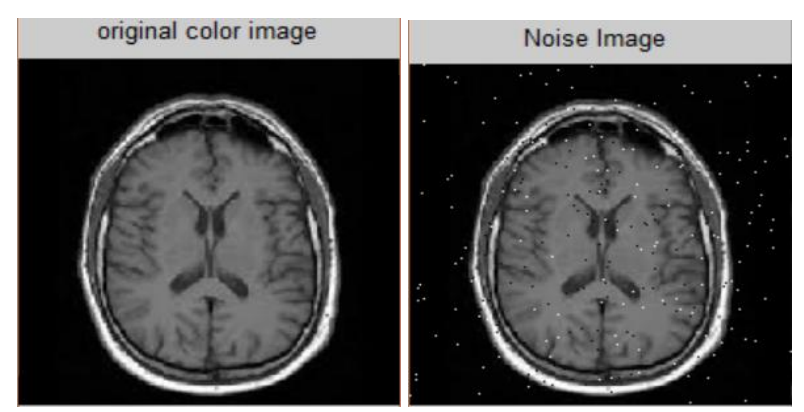

(a) Original Image

(b) 0.01 Noise Density
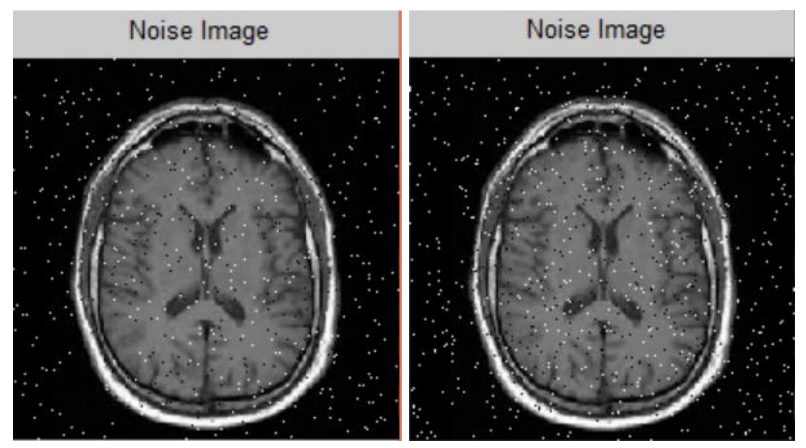

(c) 0.02 Noise Density

(d) 0.03 Noise Density
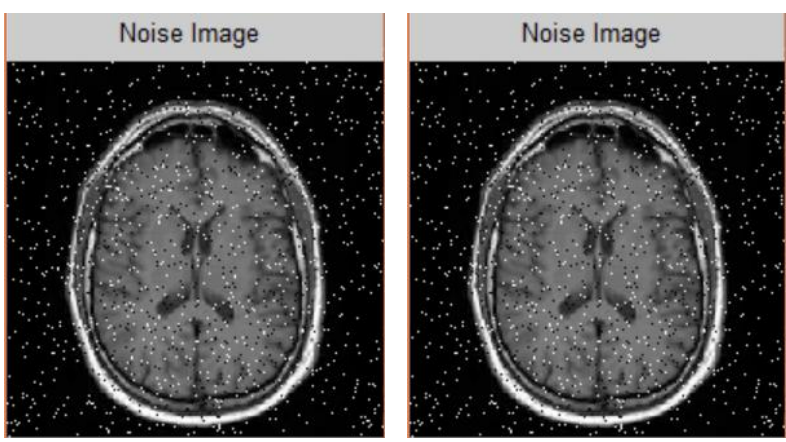

(e) 0.04 Noise Density

(f) 0.05 Noise Density

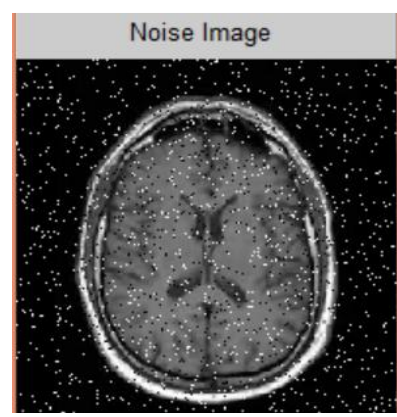

(g) 0.06 Noise Density

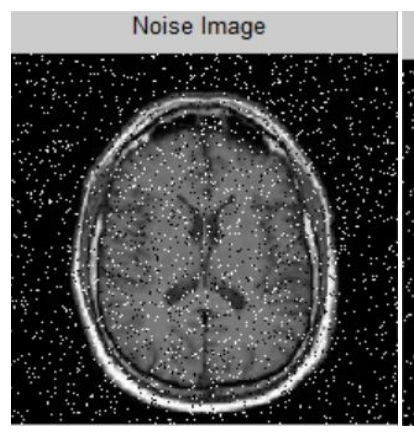

(i) 0.08 Noise Density

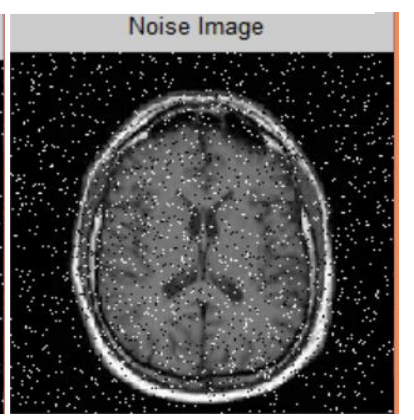

(h) 0.07 Noise Density

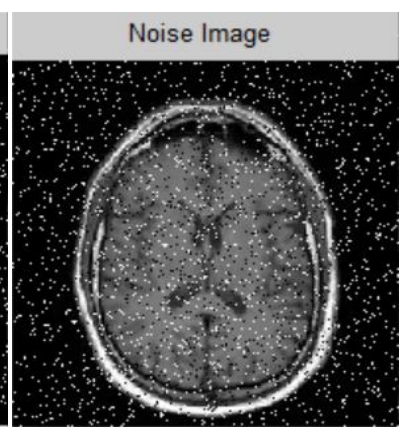

(j) 0.09 Noise Density

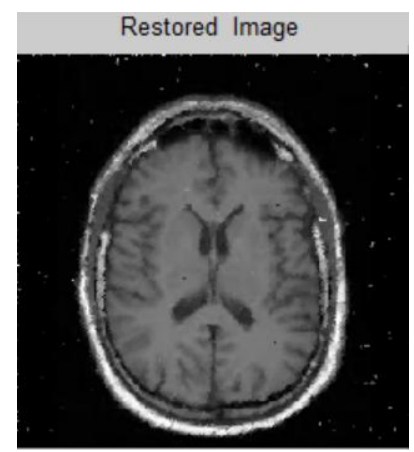

Figure 4: Experimental Salt \& Pepper Noise Image for Different Noise Density

Table 1: PSNR and MSE Value for Different Noise Density

\begin{tabular}{|c|c|c|c|c|}
\hline $\begin{array}{c}\text { Image } \\
\text { Density }\end{array}$ & NAE & MSE & RMSE & $\begin{array}{c}\text { PSNR } \\
(\mathrm{dB})\end{array}$ \\
\hline 0.01 & 0.019 & 0.126 & 0.354 & 57.177 \\
\hline 0.02 & 0.037 & 0.245 & 0.495 & 54.257 \\
\hline 0.03 & 0.057 & 0.371 & 0.608 & 52.476 \\
\hline 0.04 & 0.077 & 0.507 & 0.713 & 51.103 \\
\hline 0.05 & 0.096 & 0.633 & 0.795 & 50.153 \\
\hline 0.06 & 0.114 & 0.741 & 0.861 & 49.467 \\
\hline 0.07 & 0.136 & 0.887 & 0.942 & 48.686 \\
\hline 0.08 & 0.153 & 1.019 & 1.009 & 48.079 \\
\hline 0.09 & 0.169 & 1.107 & 1.052 & 47.722 \\
\hline
\end{tabular}

Table 2: Comparison Result

\begin{tabular}{|c|c|c|}
\hline Filter & PSNR (dB) & RMSE \\
\hline $\begin{array}{c}\text { Previous } \\
\text { Algorithm }\end{array}$ & 43.67 & 1.798 \\
\hline $\begin{array}{c}\text { Proposed } \\
\text { Algorithm }\end{array}$ & 47.722 & 1.052 \\
\hline
\end{tabular}

\section{CONCLUSION}

In this work, it can be watched that the execution of the proposed channel is better than the current channels. The fundamental commitment of the paper is a strategy that is fit for reestablishing pictures debased by speckle noise commotion with to a great degree high clamor proportion. Light is additionally tossed on the reasons for these commotions and their real sources. In the second area we introduce the different sifting systems that can be connected to de-commotion the pictures. Trial comes about displayed, demands us to finish up middle channels performed well. Adjusted middle channel is the best decision of expelling the speckle noise commotion. In this paper is utilized changed middle channel and enhanced PSNR (crest flag clamor proportion) and decreased mean square mistake (MSE) for dim and shading picture. 


\section{REFERENCES}

[1] Anitha S, Laxminarayana Kola, Sushma P and Archana S, "Analysis of Filtering and Novel Technique for Noise Removal in MRI and CT Images", International Conference on Electrical, Electronics, Communication, Computer and Optimization Techniques (ICEECCOT), IEEE 2017.

[2] Tian Bai and Jieqing Tan, "Automatic Detection and Removal of High Density Impulse Noises", IET Image Process 2015, Vol. 09, Issue 02, pp. 162-172, 2015.

[3] Priyanka Punhani and Dr. Naresh, "Noise Removal in MR Images using Non Linear Filters", 6th ICCCNT - 2015 July 13 - 15, 2015, Denton, U.S.A.

[4] Arabinda Dash and Sujaya Kumar Sathua, "High Density Noise Removal by using Cascading Algorithms", 2015 Fifth International Conference on Advanced Computing \& Communication Technologies 2327-0659/15 \$31.00 (C) 2015 IEEE.

[5] Ashutosh Pattnaik, Sharad Agarwal and Subhasis Chand. "A New and Efficient Method for Removal of High Density Salt and Pepper Noise Through Cascade Decision based Filtering Algorithm" in 2nd International Conference on Communication, Computing \& Security, Volume 6, Pages 108-117. ICCCS-2012.

[6] Chauhan, Arjun Singh, and Vineet Sahula. "High density impulsive Noise removal using decision based iterated conditional modes" in Signal Processing, Computing and
Control (ISPCC), 2015 International Conference on, pp. 24- 29. IEEE, 2015.

[7] Dash, Arabinda, and Sujaya Kumar Sathua. "High Density Noise Removal by Using Cascading Algorithms" in Advanced Computing \& Communication Technologies (ACCT), 2015 Fifth International Conference on, pp. 96101. IEEE, 2015.

[8] Esakkirajan, S., T. Veerakumar, Adabala N. Subramanyam, and Prem CH Chand. "Removal of high density salt and pepper noise through modified decision based unsymmetric trimmed median filter" in Signal Processing Letters, IEEE 18, no. 5 (2011): 287-290.

[9] Madhu S. Nair and G. Raju. "A new fuzzy-based decision algorithm for high-density impulse noise removal" in Signal, Image and Video Processing, November 2012, Volume 6, Issue 4, pp 579-595.

[10] Raza, Md Tabish, and Suraj Sawant. "High density salt and pepper noise removal through decision based partial trimmed global mean filter" in Engineering (NUiCONE), 2012 Nirma University International Conference on, pp. 15. IEEE, 2012

[11] Utaminingrum, Fitri, Keiichi Uchimura, and Gou Koutaki. "High density impulse noise removal based on linear meanmedian filter" in Frontiers of Computer Vision,(FCV), 2013 19th Korea-Japan Joint Workshop on, pp. 11-17. IEEE, 2013 